\title{
Evaluation of Intracellular Processes in Quinolinic Acid-Induced Brain Damage by Imaging Reactive Oxygen Species Generation and Mitochondrial Complex I Activity
}

Rie Hosoi ( $\square$ hosoi@sahs.med.osaka-u.ac.jp )

Osaka University https://orcid.org/0000-0002-4646-9272

Yuka Fujii

Osaka University: Osaka Daigaku

Ohba Hiroyuki

Hamamatsu Photonics K.K.

Miho Shukuri

Showa Pharmaceutical University: Showa Yakka Daigaku

\section{Shingo Nishiyama}

Hamamatsu Photonics K.K.

\section{Masakatsu Kanazawa}

Hamamatsu Photonics K.K.

Kenichiro Todoroki

University of Shizuoka

\section{Yasushi Arano}

Chiba University: Chiba Daigaku

\section{Toshihiro Sakai}

Hanwa Intelligent Medical Center

Hideo Tsukada

Hamamatsu Photonics K.K.

Osamu Inoue

Hanwa Intelligent Medical Center

\section{Research Article}

Keywords: reactive oxygen species, mitochondrial complex I, dihydroethidium, 18F-BCPP-EF, quinolinic acid, microglia

Posted Date: May 10th, 2021 
DOl: https://doi.org/10.21203/rs.3.rs-465973/v1

License: (c) (1) This work is licensed under a Creative Commons Attribution 4.0 International License. Read Full License 


\section{Abstract}

\section{Purpose}

Our study aimed to elucidate the intracellular processes associated with quinolinic acid (QA)-induced brain injury by acquiring semiquantitative fluorescent images of reactive oxygen species (ROS) generation and positron emission tomography (PET) images of mitochondrial complex I (MC-I) activity.

Methods

Ex vivo fluorescent imaging with dihydroethidium (DHE) and PET scans with ${ }^{18} \mathrm{~F}-\mathrm{BCPP}$-EF were conducted at 3 hours and 1 day after QA injection into the rat striatum. Immunohistochemical studies were performed 1 day after QA injection into the rat brain using monoclonal antibodies against neuronal nuclei (NeuN) and CD11b.

Results

A strong DHE-derived fluorescent signal was detected in a focal area within the QA-injected striatum 3 hours after QA injection, and increased fluorescent signal spread throughout the striatum and parts of the cerebral cortex after 1 day. By contrast, ${ }^{18} \mathrm{~F}$-BCPP-EF uptake in the QA-injected rat brain was unchanged after 3 hours and markedly decreased after 1 day, not only in the striatum but also in the cerebral hemisphere. The fluorescent signal in the striatum 1 day after QA injection colocalised with microglial marker expression.

\section{Conclusions}

We successfully obtained functional images of focal ROS generation during the early period of excitotoxic injury, and microglial ROS generation and mitochondrial dysfunction were observed during the progression of the inflammatory response. Both ex vivo DHE imaging and in vivo ${ }^{18} \mathrm{~F}$-BCPP-EF-PET were sufficiently sensitive to detect the respective processes of QA-induced brain damage. Our study contributes to the functional imaging of multiple events during the pathological process.

\section{Introduction}

In vivo and ex vivo brain imaging has provided useful information for the study of brain damage and dysfunction. Quantitative imaging of the living brain is useful for elucidating the processes underlying neurodegenerative diseases. The overproduction of reactive oxygen species (ROS) and mitochondrial dysfunction play important roles during the process of brain damage and dysfunction. Oxidative stress is closely associated with mitochondrial dysfunction, and mitochondria are both the major sources and primary targets of ROS. Mitochondrial complex I (MC-I), in addition to MC-III, is considered to be the primary site of ROS generation [1,2]. ROS play an important role as a regulatory mediator of signalling processes under physiological conditions, and relatively low ROS concentrations are maintained by the redox regulation system [3]. However, the persistent production of excessive ROS induces a disturbance in 
redox homeostasis. Excessive amounts of ROS cause oxidative damage to DNA, proteins, and lipids, resulting in cellular damage and cell death. Therefore, ROS and mitochondrial dysfunction have been implicated in many neurodegenerative disorders, including Alzheimer's disease; Parkinson's disease; multiple sclerosis; cerebrovascular diseases, such as ischaemia/reperfusion injury; and several psychiatric disorders [4-11].

Dihydroethidium (DHE) is a lipophilic membrane-permeable compound converted into charged membrane-impermeable products by superoxide radicals. When DHE products intercalate within the DNA, the nucleus becomes stained with a bright fluorescent red [12]. When systemically administered to animals, DHE distributes rapidly into various tissues, including the brain; if not oxidised, DHE is cleared from tissues and excreted. DHE has been widely used to evaluate ROS production in both in vitro and in vivo experiments $[13,14]$. Recently, we reported a simple procedure for imaging excessive ROS generation in intact animals utilising a planar laser scanner, Fluoro-Imaging Analyzer System (FLA-7000; Fuji Film Co, Tokyo, Japan), and a fluorescent probe, DHE. In the previous report, we successfully obtained semiquantitative fluorescent images of ROS overexpression in brain and renal disorders [15].

For quantitative imaging of $\mathrm{MC}-\mathrm{I}$ activity in the living animal brain, we previously developed a positron emission tomography (PET) probe, 2-tert-butyl-4-chloro-5-\{6-[2-(2-18 F-fluoroethoxy)-ethoxy]pyridin-3ylmethoxy\}-2H-pyridazin-3-one ( $\left({ }^{18} \mathrm{~F}-\mathrm{BCPP}-\mathrm{EF}\right)$ [16], and evaluated its ability to detect age-related reductions in MC-I activity in the brains of monkeys [17] and humans [18]; neurodegenerative damage after ischaemia-reperfusion injury in the brains of live rats [19] and monkeys [20]; and Alzheimer diseaserelated reductions in MC-I activity in the brains of monkeys [21] and humans [22]. 2-Deoxy-2-[18 $\mathrm{F}]$ fluoroglucose $\left({ }^{18} \mathrm{~F}-\mathrm{FDG}\right)$ is a commonly used probe for the quantitative measurement of glucose metabolism; however, ${ }^{18} \mathrm{~F}-\mathrm{FDG}$ is also taken up by activated inflammatory cells (microglia and macrophages) $[20,23$, 24]. In contrast, ${ }^{18} \mathrm{~F}$-BCPP-EF demonstrated reduced uptake in the regions associated with high immunoreactivity against ionised calcium-binding adaptor molecule 1 (Iba1), a microglial marker and was well-correlated with the regional cerebral metabolism of oxygen $\left(\mathrm{rCMRO}_{2}\right)$ in the ischaemic animal brain [20]. ${ }^{18} \mathrm{~F}-\mathrm{BCPP}-\mathrm{EF}$ is expected to better reflect mitochondrial dysfunction and cellular energy deficiencies in neurons than ${ }^{18} \mathrm{~F}-\mathrm{FDG}$.

In the present study, we evaluated the intracellular processes that occur in the quinolinic acid (QA)-treated rat brain: ROS generation and MC-I activity. QA is an endogenous metabolite of the kynurenine pathway that acts as an N-methyl-D-aspartate (NMDA) receptor agonist $[25,26]$. QA has a potent neurotoxic effect, and the intrastriatal injection of QA in the rat brain has been used to obtain an excitotoxic model of Huntington's disease [27-29]. During the present study, we obtained functional images of the focal ROS generation 3 hours after QA injection, and ROS generation in microglia and mitochondrial dysfunction were imaged 1 day after QA injection.

\section{Materials And Methods}


Adult male Sprague Dawley rats (8-9 weeks old) were obtained from CLEA Japan, Inc. (Tokyo, Japan). Animals were housed on a 12-hour light/dark cycle with free access to food and water. The following animal experiments were approved by the Institutional Animal Care and Use Committee, Division of Health Sciences, Graduate School of Medicine, Osaka University (Approval numbers; 31-02-2) and the Ethics Committee of the Central Research Laboratory, Hamamatsu Photonics (Approval numbers; HPK2019-07B).

2,7-Diamino-10-ethyl-9-phenyl-9,10-dihydrophenanthridine (DHE) was obtained from AdipoGen AG (Liestal, Switzerland). 2,3-Pyridinedicarboxylic acid (QA) was obtained from Nacalai Tesque Inc. (Kyoto, Japan). The precursor of 2-tert-butyl-4-chloro-5-\{6-[2-(2-18F-fluoroethoxy)-ethoxy]pyridin-3-ylmethoxy\}-2Hpyridazin-3-one ( $\left.{ }^{18} \mathrm{~F}-\mathrm{BCPP}-\mathrm{EF}\right)$ and its corresponding cold compounds were purchased from NARD Institute (Amagasaki, Japan). The synthesis of ${ }^{18} \mathrm{~F}-\mathrm{BCPP}$-EF was performed as described previously [16]. All other chemicals utilised in this study were the highest grade commercially available.

Surgery and microinjection of QA

Surgery preparation and QA injections were performed according to previously described protocols [3033]. For the implantation of guide cannulas into the striatum, the animal was anaesthetised with isoflurane (induction $5 \%$, maintenance $2 \%$ ). Next, the head of the animal was placed in a stereotaxic apparatus. Bilateral 26-gauge stainless steel guide cannulas fitted with 30-gauge stainless steel obturators were placed into the striatum at the following position: $3.2 \mathrm{~mm}$ lateral to the midline, $0.2 \mathrm{~mm}$ anterior to Bregma, and $2.0 \mathrm{~mm}$ below the cortical surface [34]. The guide cannulas were then fixed to the skull with acrylic cement and two stainless steel screws. After the surgery, the animals were allowed to recover for several days.

QA (60 nmol/ $\mu \mathrm{l})$ was dissolved in saline, and the drug solution was injected $(0.2 \mu \mathrm{l} / \mathrm{min}, 10$ minutes) through a 30-gauge cannula ( $5.5 \mathrm{~mm}$ below the cortical surface) into the left striatum of each rat while the rat was awake. The injection cannula was left in place for an additional 3 minutes to reduce the reflux of the injected chemicals along the cannula track. Simultaneously, saline solution was injected through a cannula (30-gauge, $5.5 \mathrm{~mm}$ below the cortical surface) into the right striatum. Subsequently, animals were subjected to ex vivo DHE experiments and PET measurements using ${ }^{18} \mathrm{~F}-\mathrm{BCPP}$-EF.

DHE administration and ROS detection with the FLA-7000

DHE ( $5 \mathrm{mg} / \mathrm{kg}$ ) was administered through the tail vein 3 hours and 1 day after the QA injection. The animals were then sacrificed 60 minutes after the injection of DHE by decapitation under brief anaesthesia. The brains were quickly removed and frozen. Next, coronal slices (20- $\mu \mathrm{m}$-thick) were prepared using a cryostat at $-20^{\circ} \mathrm{C}$ and placed onto glass slides. The brain slices were scanned using a $532 \mathrm{~nm}$ excitation laser and a $580 \mathrm{~nm}$ long-pass detection filter with a Fluoro-Imaging Analyzer System (FLA-7000; Fuji Film Co., Tokyo, Japan). The fluorescence intensity in the brain slices was measured in 
linear arbitrary units (LAU), corrected for background ([LAU-background] / area [mm²]) with Multi-Gauge Analysis Software (Fuji Film Co., Tokyo, Japan). The background value was obtained as the fluorescence intensity of a region of the glass slide containing no brain slices.

PET imaging

${ }^{18} \mathrm{~F}-\mathrm{BCPP}$-EF (58.5-66.7 MBq/kg animal, iv) was injected 3 hours and 1 day after the QA injection. The PET scans were conducted with a high-resolution animal PET scanner (SHR-38000; Hamamatsu Photonics, Hamamatsu, Japan) under isoflurane anaesthesia. Dynamic images and summation images

of ${ }^{18} \mathrm{~F}-\mathrm{BCPP}-\mathrm{EF}$, from 0 to 90 minutes after the injection, were reconstructed and used to create standardised uptake value (SUV) images. The body temperatures of the rats were monitored and maintained using a heating pad during PET measurement. Volumes of interest (VOIs) were placed on PET images in the striatum, prefrontal cortex, frontal cortex, parietal cortex, hippocampus, thalamus, and cerebellum, with the aid of Waxholm Space Atlas of the Sprague Dawley Rat Brain (the INCF Software Center, https://www.nitrc.org/projects/whs-sd-atlas) and rat brain atlas [34].

Histochemistry

The frozen sections used in the ex vivo DHE experiments were stained with Cresyl violet. The stained sections were then visualised using a Nikon Eclipse 80i microscope (Nikon Co., Tokyo, Japan), and the images were captured using the NIS-Elements BR 2.30 software (Nikon Co., Tokyo, Japan).

For the immunohistochemical study, the rats were deeply anaesthetised and perfused transcardially with ice-cold saline, followed by $4 \%$ paraformaldehyde solution, 1 day after QA injection. The brains were removed, post-fixed with paraformaldehyde solution for 6 hours, placed in a $30 \%$ sucrose solution, and embedded in optimal cutting temperature (OCT) compound (Sakura Finetek Japan Co., Ltd., Tokyo,

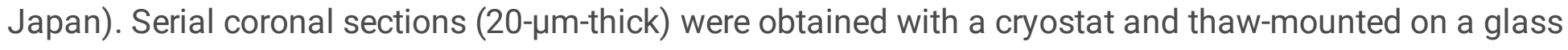
slide. The antibodies used in this study were as follows: mouse monoclonal anti-neuron-specific nuclear protein (NeuN, 1:500, Merck Millipore Co., Darmstadt, Germany), and mouse monoclonal anti-CD11b antibody (clone OX-42, 1:500, AbD Serotec, Oxfordshire, UK). After the overnight incubation of the brain sections with the antibodies at room temperature, the primary antibodies were visualised with Cy2labelled secondary antibody (1:500, Jackson ImmunoResearch, Newmarket, UK). Fluorescent images were captured with a 3- laser confocal microscope (Nikon Co., Tokyo, Japan).

\section{Statistical analysis}

All values are expressed as the mean \pm SD (for each group). Differences were assessed with Student's paired t-tests. P-values less than 0.05 were considered significant.

\section{Results}


A typical distribution of QA-induced fluorescent signal in the rat brain slices at the striatal level is shown in Figure 1A. A focal strong fluorescent signal in a small area and a slight increase in fluorescent signal throughout the striatum were detected in the QA-injected side 3 hours after the QA injection, and the strong fluorescent signal had spread throughout the entire striatum by 1 day after QA injection (Fig. 1A and $B)$. A marked increase in the fluorescent signal was also detected in specific regions of the cerebral cortex 1 day after QA injection, as shown in the right panel of Figure $1 \mathrm{~A}$.

For the evaluation of QA injections effects on MC-I activity in the brain, we obtained PET images using ${ }^{18} \mathrm{~F}-\mathrm{BCPP}$-EF at 3 hours and 1 day after QA injection (Fig. 2A). Three hours after QA injection, no significant differences in ${ }^{18} \mathrm{~F}$-BCPP-EF uptake were observed between saline- and QA-injected brain hemispheres. By contrast, 1 day after QA injection, ${ }^{18} \mathrm{~F}-\mathrm{BCPP}$-EF uptake was significantly decreased by $30.9 \%$ compared with that on the saline-injected side (Fig. 2B) throughout the whole striatum.

The fluorescence signals from DHE-injected rats tended to increase in the prefrontal cortex 1 day after QA injection, but no changes were observed in other regions (Fig. 3A and C). By contrast, ${ }^{18} \mathrm{~F}-\mathrm{BCPP}$-EF uptake 1 day after QA injection was reduced not only in the striatum but also in cerebral regions. Thus, ${ }^{18} \mathrm{~F}-\mathrm{BCPP}$ EF uptake was significantly reduced in the prefrontal cortex and thalamus. Although no significant difference was observed in the frontal cortex, parietal cortex, and hippocampus, a tendency towards reduced ${ }^{18} \mathrm{~F}$-BCPP-EF uptake was observed following QA injection (Fig. 3B and D), indicating that the QAinduced impairment of $\mathrm{MC}-\mathrm{I}$ activity expanded from the injection point into surrounding areas. When comparing the extent of QA influence 1 day after injection between the DHE-derived fluorescent signal and ${ }^{18} \mathrm{~F}$-BCPP-EF uptake, we found that ${ }^{18} \mathrm{~F}$-BCPP-EF uptake was more strongly affected by QA over a larger area of the cerebral hemisphere.

Nissl staining of brain slices was performed after the acquisition of ex vivo fluorescent images, 3 hours after QA injection, which showed remarkable neuronal degradation in the striatum at the site of QA injection (Fig. 4B), although in a narrower area than the area associated with a strong fluorescence signal (Fig. 1A). This neuronal degradation was localised within an approximately 1.0-mm-diameter area (Fig. 4B), and neurons a few mm away from the injection site remained intact (Fig. 4C). One day after QA injection, the neuronal degradation had spread throughout the entire striatum (Fig. 4E), but no abnormalities were observed in the prefrontal cortex (Supp. Fig. 1B) and thalamus (Supp. Fig. 1D).

To clarify which cell types were producing DHE-derived fluorescence signals, immunohistochemical studies were performed in the striatum 1 day after QA injection, using monoclonal antibodies against NeuN, a marker for neurons (Fig. 5A-F), and CD11b, a marker for activated microglia (Fig. 5G-L). In the QA-injected striatum, the quantity of NeuN-positive cells was reduced (Fig. 5E) compared with that in the saline-injected striatum (Fig. 5B), and CD11b-positive cells were increased (Fig. 5K) compared with those in the saline-injected striatum (Fig. $5 \mathrm{H}$ ). Furthermore, as shown in Figure $5 \mathrm{~L}$, the majority of DHE fluorescence signals colocalised with CD11b-positive cells in the QA-injected striatum.

\section{Discussion}


QA is an endogenous analogue of glutamate that acts as an NMDA receptor agonist [25,26]. QA is an excitotoxic metabolite of the kynurenine pathway with high in vivo potency as a neurotoxin. QA administration increases the levels of extracellular glutamate $[35,36]$ and increases cytosolic $\mathrm{Ca}^{2+}$ concentrations in both neurons and astrocytes. Furthermore, QA excitotoxicity is associated with its ability to increase lipid peroxidation [37] and induce free radical generation [38], which consequently induces oxidative stress and energetic dysfunction. Intrastriatal QA injections induce excitotoxicity in animals [39], resulting in neuronal lesions [28].

In the present study, increased ROS generation, as imaged using a fluorescent signal, was detected in a focal area within the striatum as early as 3 hours after QA injection, and this signal spread throughout the striatum and parts of the cerebral cortex 1-day post-injection. At 3 hours after QA injection, the area of neuronal degradation in the striatum was observed to be smaller than the area of excessive ROS generation. At 1 day after QA injection, the ROS-associated fluorescent signal was primarily detected in microglia in the QA-injected striatum. Although ${ }^{18} \mathrm{~F}$-BCPP-EF uptake was unchanged 3 hours after QA injection, a marked decrease in uptake was observed after 1 day, not only in the striatum but also in the cerebral hemisphere, indicating that the delayed impairment of MC-I activity was more extensive than ROS generation 1 day after QA injection.

The focal ROS generation observed 3 hours after QA injection may reflect the mitochondrial ROS generation induced by excessive $\mathrm{Ca}^{2+}$ influx, mediated by NMDA receptor activation or represent ROS generated by the Fenton reaction between QA-Fe ${ }^{2+}$ complexes [40]. In our preliminary study, a similar degree of focal ROS generation was observed as early as 1 hour after QA injection (data not shown). Santamaría et al. have shown that transient increases in ROS generation associated with the overactivation of NMDA receptors and other mechanisms occur as early as 1 hour after QA infusion into the rat striatum in vivo [41]. Although several other reports have shown that QA injections modify ROS generation at later time points after QA injections into animal brains [42, 43], a large amount of ROS generation appears to occur in a restricted area, and a mild increase in ROS generation appears to occur throughout the striatum.

The present microscopic analyses of combined fluorescence signals and immunohistochemical staining demonstrated that explosive ROS generation occurred in microglia located within the striatum 1 day after QA injection. At this time point, neuronal degradation and microglial activation were detected in the striatum. Neuronal damage caused by excitotoxic factors has been reported to stimulate a proinflammatory cascade. In this cascade, microglial activation occurs in response to neuronal excitotoxic injury, which, in turn, activates microglia to release ROS and neurotoxic cytokines $[44,45,46]$. This cascade results in a self-propelled, progressive cycle of microglial activation and neuronal damage. Under these conditions, excessive ROS generation in the microglia may lead to an oxidant/antioxidant imbalance, in which the antioxidative response may not be sufficient to normalise redox homeostasis. The ROS generation by microglia can be originated from several sources; however, NADPH oxidase activation has been reported to enhance the release of other proinflammatory factors from microglia [47, 48], and this enzyme might represent an important factor in microglia-mediated neurotoxicity. Oxidative 
stress is well known to be associated with inflammation and disease onset; thus, ROS generation in microglia may serve as a valuable target with therapeutic benefits for the treatment of neuronal disorders.

In the present study, ${ }^{18} \mathrm{~F}$-BCPP-EF uptake in the QA-injected rat brain was unchanged after 3 hours, indicating that $\mathrm{MC}-\mathrm{I}$ activity remained intact during this early period after QA injection. Bordelon et al. reported progressive mitochondrial dysfunction in the rat striatum 12 hours after QA injection [49]. Ribeiro et al. also reported reduced activity in MC-II and MC-III in the respiratory chain in the striatum 12 hours after QA injection but not 3 or 6 hours after QA injection [50]. These results, together with those of the present study, indicate that mitochondrial proteins or their functions may survive through at least the early period after QA-induced excitotoxic injury.

A decrease in MC-I activity 1 day after QA injection could be observed not only in the striatum, where neuronal degradation occurred, but also in the cerebral hemisphere, where cells remained intact. In the QA-injected striatum, decreased MC-I activity may reflect mitochondrial dysfunction in the proinflammatory cascade and the self-propelled, progressive cycle of microglial activation and neuronal degradation. Furthermore, the inhibition of MC-I has been reported to induce robust ROS generation from the mitochondria, which activates NLR family pyrin domain-containing 3 (NLRP3) inflammasome [51, 52], driving several inflammatory processes and mediating the release of interleukin (IL)-1 family cytokines [53]. Thus, ${ }^{18} \mathrm{~F}$-BCPP-EF appears to serve as an excellent probe that reflects neuronal mitochondrial dysfunction during the proinflammatory cascade.

$\mathrm{MC}-\mathrm{I}$ activity, as measured using ${ }^{18} \mathrm{~F}-\mathrm{BCPP}$-EF, was reduced in areas where cells remained intact 1 day after QA injection. Brain damage often causes changes in cellular metabolism and function, not only near the neural degradation area but also in more remote brain regions that are functionally and anatomically connected to the injured area. When QA is injected into the striatum of adult rats, neurogenesis has been reported to occur between 1 and 14 days after injection [54,55]. One possibility is that neurogenesis shifts the balance of energy production from mitochondria-related oxidative metabolism to glycolysis in the cerebral hemisphere on the QA-injected side. Alternatively, the inhibition of MC-I activity has been reported to activate microglia [56-58]. Another study suggested that mitochondrial dysfunction in microglia exacerbated the proinflammatory M1 phenotype and increased ROS production [59]. In addition, the removal of impaired or dysfunctional mitochondria has been reported to be neuroprotective [60]. Thus, QA injection into the striatum has been speculated to stimulate neurogenesis or cause more severe and extensive damage to the cerebral hemisphere on the QA-injected side during later phases. Based on the results of the present study, whether the observed decrease in MC-I activity is temporary due to a defensive cellular response or is long-lasting, associated with subsequent cellular damage, is not known. Whether reduced MC-I activity counteracts or exacerbates pathological effects is also unclear. These questions are important subjects for future research.

In this study, multiple molecular probes were used to image and monitor the intracellular neurodegenerative processes. This approach is useful for understanding brain function and disease and could contribute to disease diagnosis, therapeutic development, and the assessment of therapeutic 
effects. In the brain, functional imaging, including neuron-microglia, or neuron-astrocyte interactions, is also necessary. In the present study, we used ex vivo imaging to perform ROS detection. For in vivo imaging, we recently developed a novel PET probe, ${ }^{18} \mathrm{~F}$-labelled dihydromethidine, to detect ROS [61]. Multi-tracer imaging in the same animals using PET probes is expected in future studies.

\section{Conclusion}

We successfully obtained functional images of focal ROS generation during the early period of excitotoxic injury, and microglial ROS generation and mitochondrial dysfunction were observed during the progression of the inflammatory response. Both ex vivo DHE imaging and in vivo ${ }^{18} \mathrm{~F}$-BCPP-EF-PET were sufficiently sensitive to detect the respective processes of QA-induced brain damage. Our study contributes to the functional imaging toolbox available to monitor multiple events in the pathological process.

\section{Declarations}

Ethics approval and consent to participate

All animal studies were approved by the Institutional Animal Care and Use Committee, Division of Health Sciences, Graduate School of Medicine, Osaka University and the Ethics Committee of the Central Research Laboratory, Hamamatsu Photonics. All applicable international, national, and/or institutional guidelines for the care and use of animals were followed.

The authors have agreed to participate in the paper.

Consent for publication

The authors have agreed to transfer copyright to European Journal of Nuclear Medicine and Molecular Imaging in the event that this manuscript is accepted for publication.

Availability of data and material

The data that support the findings of this study are available from the corresponding author, R-hosoi, upon reasonable request.

Competing interests

The authors declare that they have no competing of interest.

Funding

Not applicable

Authors' contributions 
All authors contributed to the study conception and design. Material preparation, data collection and analysis were performed by Rie Hosoi, Yuka Fujii, Ohba Hiroyuki, Miho Shukuri, Shingo Nishiyama, Masakatsu Kanazawa, and Hideo Tsukada. The first draft of the manuscript was written by Rie Hosoi and all authors commented on previous versions of the manuscript. All authors read and approved the final manuscript.

Acknowledgements

Not applicable

\section{References}

[1] Kowaltowski AJ, de Souza-Pinto NC, Castilho RF and Vercesi AE. Mitochondria and reactive oxygen species. Free Radic Biol Med. 2009;47(4):333-43. doi: 10.1016/j.freeradbiomed.2009.05.004

[2] Brand MD. The sites and topology of mitochondrial superoxide production. Exp Gerontol. 2010;45(78):466-72. doi: 10.1016/j.exger.2010.01.003.

[3] Dröge W. Free radicals in the physiological control of cell function. Physiol Rev. 2002; 82(1):47-95. doi: 10.1152/physrev.00018.2001

[4] Maier $\mathrm{CM}$, Chan $\mathrm{PH}$. Role of superoxide dismutases in oxidative damage and neurodegenerative disorders. Neuroscientist. 2002;8(4):323-34.

[5] Wang X, Lo EH. Triggers and mediators of hemorrhagic transformation in cerebral ischemia. Mol Neurobiol. 2003;28(3):229-44. doi: 10.1385/MN:28:3:229

[6] Behrens MM, Ali SS, Dao DN, Lucero J, Shekhtman G, Quick KL, Dugan LL. Ketamine-induced loss of phenotype of fast-spiking interneurons is mediated by NADPH-oxidase. Science. 2007;318(5856):1645-7. doi: 10.1126/science. 1148045

[7] Dugan LL, Ali SS, Shekhtman G, Roberts AJ, Lucero J, Quick KL, Behrens MM. IL-6 mediated degeneration of forebrain GABAergic interneurons and cognitive impairment in aged mice through activation of neuronal NADPH oxidase. PLoS One. 2009;4(5):e5518. doi: 10.1371/journal.pone.0005518 [8] Hirsch EC, Hunot S. Neuroinflammation in Parkinson's disease: a target for neuroprotection? Lancet Neurol. 2009;8(4):382-97. doi: 10.1016/S1474-4422(09)70062-6

[9] Drechsel DA, Estévez AG, Barbeito L, Beckman JS. Nitric oxide-mediated oxidative damage and the progressive demise of motor neurons in ALS. Neurotox Res. 2012;22(4):251-64. doi: 10.1007/s12640012-9322-y

[10] Popa-Wagner A, Mitran S, Sivanesan S, Chang E, Buga AM. ROS and brain diseases: the good, the bad, and the ugly. Oxid Med Cell Longev. 2013;963520. doi: 10.1155/2013/963520 
[11] Ayala, A, Venero, JL, Cano, J, Machado, A. Mitochondrial toxins and neurodegenerative diseases. Front. Biosci. 2007;12:986-1007. doi: 10.2741/2119

[12] Bucana C, Saiki I, Nayar R. Uptake and accumulation of the vital dye hydroethidine in neoplastic cells. J Histochem Cytochem. 1986;34(9):1109-15.

[13] Bindokas VP, Jordán J, Lee CC, Miller RJ. Superoxide production in rat hippocampal neurons: selective imaging with hydroethidine. J Neurosci. 1996;16(4):1324-36.

[14] Hall DJ, Han SH, Chepetan A, Inui EG, Rogers M, Dugan LL. Dynamic optical imaging of metabolic and NADPH oxidase-derived superoxide in live mouse brain using fluorescence lifetime unmixing. J Cereb Blood Flow Metab. 2012;32(1):23-32. doi: 10.1038/jcbfm.2011.119.

[15] Hosoi R, Sato S, Shukuri M, Fujii Y, Todoroki K, Arano Y, Sakai T, Inoue O. A Simple Ex Vivo Semiquantitative Fluorescent Imaging Utilizing Planar Laser Scanner: Detection of Reactive Oxygen Species Generation in Mouse Brain and Kidney. Mol Imaging. 2019;18:1536012118820421. doi: $10.1177 / 1536012118820421$.

[16] Tsukada H, Nishiyama S, Fukumoto D, Kanazawa M, Harada N. Novel PET probes ${ }^{18} \mathrm{~F}-\mathrm{BCPP}$-EF and ${ }^{18} \mathrm{~F}-\mathrm{BCPP}-\mathrm{BF}$ for mitochondrial complex I: A PET study in comparison with ${ }^{18} \mathrm{~F}-\mathrm{BMS}-747158-02$ in rat brain. J Nucl Med. 2014;55:473-480.

[17] Tsukada H, Ohba H, Kanazawa M, Kakiuchi T, Harada N. Evaluation of ${ }^{18} \mathrm{~F}-\mathrm{BCPP}-\mathrm{EF}$ for mitochondrial complex 1 imaging in conscious monkey brain using PET. Eur J Nucl Med Mol Imaging. 2014;41(4):75563. doi: 10.1007/s00259-013-2628-z.

[18] Mansur A, Rabiner EA, Comley RA, Lewis Y, Middleton LT, Huiban M, et al. Characterization of 3 PET tracers for quantification of mitochondrial and synaptic function in healthy human brain: ${ }^{18} \mathrm{~F}-\mathrm{BCPP}-\mathrm{EF}$, ${ }^{11}$ C-SA-4503, ${ }^{11}$ C-UCB-J. J Nucl Med. 2020;61:96-103.

[19] Fukuta T, Asai T, Ishii T, Koide H, Kiyokawa C, Hashimoto M, et al. Non-invasive evaluation of neuroprotective drug candidates for cerebral infarction by PET imaging of mitochondrial complex-I activity. Sci Rep. 2016;6:30127.

[20] Tsukada H, Ohba H, Nishiyama S, Kanazawa M, Kakiuchi T, Harada N. PET imaging of ischemiainduced impairment of mitochondrial complex I function in monkey brain. J Cereb Blood Flow Metab. 2014;34(4):708-14. doi: 10.1038/jcbfm.2014.5.

[21] Tsukada H, Nishiyama S, Ohba H, Kanazawa M, Kakiuchi T, Harada N. Comparing amyloid-b deposition, neuroinflammation, glucose metabolism, and mitochondrial complex I activity in brain: A PET study in aged monkeys. Eur J Nucl Med Mol Imaging. 2014;41:2127-2136. 
[22] Terada T, Obi T, Bunai T, Matsudaira T, Yoshiksawa E, Ando I, et al. In vivo mitochondrial and glycolytic impairments in Alzheimer's disease. Neurology. 2020; 94:e1592-e1604.

[23] Kubota R, Kubota K, Yamada S, Tada M, Ido T, Tamahashi N. Microautoradiographic study for differentiation of intratumoral macrophages, granulation tissue and cancer cells by the dynamic fluoro18-fluorodeoxyglucose uptake. J Nucl Med. 1994;35:104-112.

[24] Tsukada H, Sato K, Fukumoto D, Nishiyama S, Harada N, Kakiuchi T. Evaluation of D-isomers of O${ }^{11} \mathrm{C}$-methyl tyrosine and $\mathrm{O}-{ }^{18} \mathrm{~F}$-fluoromethyl tyrosine, as tumor imaging agents in tumor-bearing mice: a comparison study with L- and D- ${ }^{11}$ C-methionine. J Nucl Med. 2006;47:679-688.

[25] Stone TW, Perkins MN. Quinolinic acid: a potent endogenous excitant at amino acid receptors in CNS.Eur J Pharmacol. 1981;72(4):411-2.

[26] Stone TW. Neuropharmacology of quinolinic and kynurenic acids. Pharmacol Rev. 1993;45(3):30979.

[27] Schwarcz R, Köhler C. Differential vulnerability of central neurons of the rat to quinolinic acid. Neurosci Lett. 1983;38(1):85-90.

[28] Schwarcz R, Whetsell WO Jr, Mangano RM. Quinolinic acid: an endogenous metabolite that produces axon-sparing lesions in rat brain. Science. 1983;219(4582):316-8.

[29] Beal MF, Kowall NW, Ellison DW, Mazurek MF, Swartz KJ, Martin JB. Replication of the neurochemical characteristics of Huntington's disease by quinolinic acid. Nature. 1986;321(6066):168-71. doi:

$10.1038 / 321168 \mathrm{a} 0$

[30] Inoue O, Yanamoto K, Fujiwara Y, Hosoi R, Kobayashi K, Tsukada H. Sensitivities of benzodiazepine receptor binding and muscarinic acetylcholine receptor binding for the detection of neural cell death caused by sodium nitroprusside microinjection in rat brain. Synapse. 2003;49(2):134-41. doi:

$10.1002 /$ syn. 10217

[31] Yanamoto K, Hosoi R, Uesaka Y, Abe K, Tsukada H, Inoue O. Intrastriatal microinjection of sodium nitroprusside induces cell death and reduces binding of dopaminergic receptors. Synapse. 2003;50(2):137-43. doi: 10.1002/syn.10256

[32] Hosoi R, Okada M, Hatazawa J, Gee A, Inoue O. Effect of astrocytic energy metabolism depressant on ${ }^{14} \mathrm{C}$-acetate uptake in intact rat brain. J Cereb Blood Flow Metab. 2004 ;24(2):188-90. doi: 10.1097/01.WCB.0000098606.42140.02

[33] Hosoi R, Matsumura A, Mizokawa S, Tanaka M, Nakamura F, Kobayashi K, Watanabe Y, Inoue 0. MicroPET detection of enhanced ${ }^{18} \mathrm{~F}$-FDG utilization by PKA inhibitor in awake rat brain. Brain Res. 2005;1039(1-2):199-202. doi: 10.1016/j.brainres.2005.01.064 
[34] Paxinos G, Watson C. The Rat Brain in stereotaxic coordinates. 4th edition. San Diego: Academic Press; 1998.

[35] Tavares RG, Tasca Cl, Santos CE, Wajner M, Souza DO, Dutra-Filho CS. Quinolinic acid inhibits glutamate uptake into synaptic vesicles from rat brain. Neuroreport. 2000;11(2):249-53.

[36] Tavares RG, Tasca Cl, Santos CE, Alves LB, Porciúncula LO, Emanuelli T, Souza DO. Quinolinic acid stimulates synaptosomal glutamate release and inhibits glutamate uptake into astrocytes. Neurochem Int. 2002;40(7):621-7.

[37] Santamaría A, Ríos C. MK-801, an N-methyl-D-aspartate receptor antagonist, blocks quinolinic acidinduced lipid peroxidation in rat corpus striatum. Neurosci Lett. 1993;159(1-2):51-4.

[38] Ganzella M, Jardim FM, Boeck CR, Vendite D. Time course of oxidative events in the hippocampus following intracerebroventricular infusion of quinolinic acid in mice. Neurosci Res. 2006;55(4):397-402. doi: 10.1016/j.neures.2006.05.003

[39] Foster AC, Collins JF, Schwarcz R. On the excitotoxic properties of quinolinic acid, 2,3-piperidine dicarboxylic acids and structurally related compounds. Neuropharmacology. 1983 ;22(12A):1331-42.

[40] Iwahashi H, Kawamori H, Fukushima K. Quinolinic acid, alpha-picolinic acid, fusaric acid, and 2,6pyridinedicarboxylic acid enhance the Fenton reaction in phosphate buffer. Chem Biol Interact. 1999;118(3):201-15. doi: 10.1016/s0009-2797(99)00080-0.

[41] Santamaría A, Jiménez-Capdeville ME, Camacho A, Rodríguez-Martínez E, Flores A, Galván-Arzate S. In vivo hydroxyl radical formation after quinolinic acid infusion into rat corpus striatum. Neuroreport. 2001;12(12):2693-6.

[42] Ganzella M, Jardim FM, Boeck CR, Vendite D. Time course of oxidative events in the hippocampus following intracerebroventricular infusion of quinolinic acid in mice. Neurosci Res. 2006;55(4):397-402. doi: 10.1016/j.neures.2006.05.003

[43] Tronel C, Rochefort GY, Arlicot N, Bodard S, Chalon S, Antier D. Oxidative stress is related to the deleterious effects of heme oxygenase- 1 in an in vivo neuroinflammatory rat model. Oxid Med Cell Longev. 2013;264935. doi: 10.1155/2013/264935.

[44] Block ML, Hong JS. Chronic microglial activation and progressive dopaminergic neurotoxicity. Biochem Soc Trans. 2007;35(Pt 5):1127-32. doi: 10.1042/BST0351127.

[45] Block ML, Hong JS. Microglia and inflammation-mediated neurodegeneration: multiple triggers with a common mechanism. Prog Neurobiol. 2005;76(2):77-98. doi: 10.1016/j.pneurobio.2005.06.004

[46] Ano Y, Sakudo A, Kimata T, Uraki R, Sugiura K, Onodera T. Oxidative damage to neurons caused by the induction of microglial NADPH oxidase in encephalomyocarditis virus infection. Neurosci Lett. 
2010;469(1):39-43. doi: 10.1016/j.neulet.2009.11.040.

[47] Qin L, Liu Y, Wang T, Wei SJ, Block ML, Wilson B, Liu B, Hong JS. NADPH oxidase mediates lipopolysaccharide-induced neurotoxicity and proinflammatory gene expression in activated microglia. $\mathrm{J}$ Biol Chem 2004;279(2):1415-21. doi: 10.1074/jbc.M307657200. Epub 2003 Oct 24.

[48] Roy A, Jana A, Yatish K, Freidt MB, Fung YK, Martinson JA, Pahan K. Reactive oxygen species upregulate $\mathrm{CD} 11 \mathrm{~b}$ in microglia via nitric oxide: Implications for neurodegenerative diseases. Free Radic Biol Med 2008; ;45(5):686-99. doi: 10.1016/j.freeradbiomed.2008.05.026. Epub 2008 Jun 6.

[49] Bordelon YM, Chesselet MF, Nelson D, Welsh F, Erecińska M. Energetic dysfunction in quinolinic acidlesioned rat striatum. J Neurochem. 1997;69(4):1629-39. doi: 10.1046/j.1471-4159.1997.69041629.x.

[50] Ribeiro CA, Grando V, Dutra Filho CS, Wannmacher CM, Wajner M. Evidence that quinolinic acid severely impairs energy metabolism through activation of NMDA receptors in striatum from developing rats. J Neurochem. 2006;99(6):1531-42. doi: 10.1111/j.1471-4159.2006.04199.x.

[51] Chen Q, Vazquez EJ, Moghaddas S, Hoppel CL, Lesnefsky EJ. Production of reactive oxygen species by mitochondria: central role of complex III. J Biol Chem. 2003;278(38):36027-31. doi: 10.1074/jbc.M304854200. Epub 2003 Jul 2.

[52] Zhou R, Yazdi AS, Menu P, Tschopp J. A role for mitochondria in NLRP3 inflammasome activation. Nature. 2011;469(7329):221-5. doi: 10.1038/nature09663. Epub 2010 Dec 1.

[53] Swanson KV, Deng M, Ting JP. The NLRP3 inflammasome: molecular activation and regulation to therapeutics. Nat Rev Immunol. 2019 Aug;19(8):477-489. doi: 10.1038/s41577-019-0165-0.

[54] Tattersfield AS, Croon RJ, Liu YW, Kells AP, Faull RL, Connor B. Neurogenesis in the striatum of the quinolinic acid lesion model of Huntington's disease. Neuroscience. 2004;127(2):319-32. doi: 10.1016/j.neuroscience.2004.04.061.

[55] Gordon RJ, Tattersfield AS, Vazey EM, Kells AP, McGregor AL, Hughes SM, Connor B. Temporal profile of subventricular zone progenitor cell migration following quinolinic acid-induced striatal cell loss. Neuroscience. 2007 Jun 8;146(4):1704-18. doi: 10.1016/j.neuroscience.2007.03.011

[56] Shaikh SB, Nicholson LF. Effects of chronic low dose rotenone treatment on human microglial cells. Mol Neurodegener. 2009;4:55. doi: 10.1186/1750-1326-4-55.

[57] Yuan YH, Sun JD, Wu MM, Hu JF, Peng SY, Chen NH. Rotenone could activate microglia through NFkappaB associated pathway. Neurochem Res. 2013;38:1553-60. DOI: 10.1007/s11064-013-1055-7

[58] Ye J, Jiang Z, Chen X, Liu M, Li J, Liu N. Electron transport chain inhibitors induce microglia activation through enhancing mitochondrial reactive oxygen species production. Exp Cell Res. 2016;340:315-26. DOI: 10.1016/j.yexcr.2015.10.026 
[59] Tang Y, Le W. Differential Roles of M1 and M2 Microglia in Neurodegenerative Diseases. Mol Neurobiol. 2016;53:1181-1194. DOI: 10.1007/s12035-014-9070-5

[60] Loeb LA, Wallace DC, Martin GM. The mitochondrial theory of aging and its relationship to reactive oxygen species damage and somatic mtDNA mutations. Proc Natl Acad Sci U S A. 2005; 102(52):1876918770. doi: 10.1073/pnas.0509776102

[61] Egami H, Nakagawa S, Katsura Y, Kanazawa M, Nishiyama S, Sakai T, Arano Y, Tsukada H, Inoue O, Todoroki K, Hamashima Y. 18F-Labeled dihydromethidine: positron emission tomography radiotracer for imaging of reactive oxygen species in intact brain. Org. Biomol. Chem., 2020;18:2387-2391. DOI: 10.1039/D00B00126K

\section{Figures}
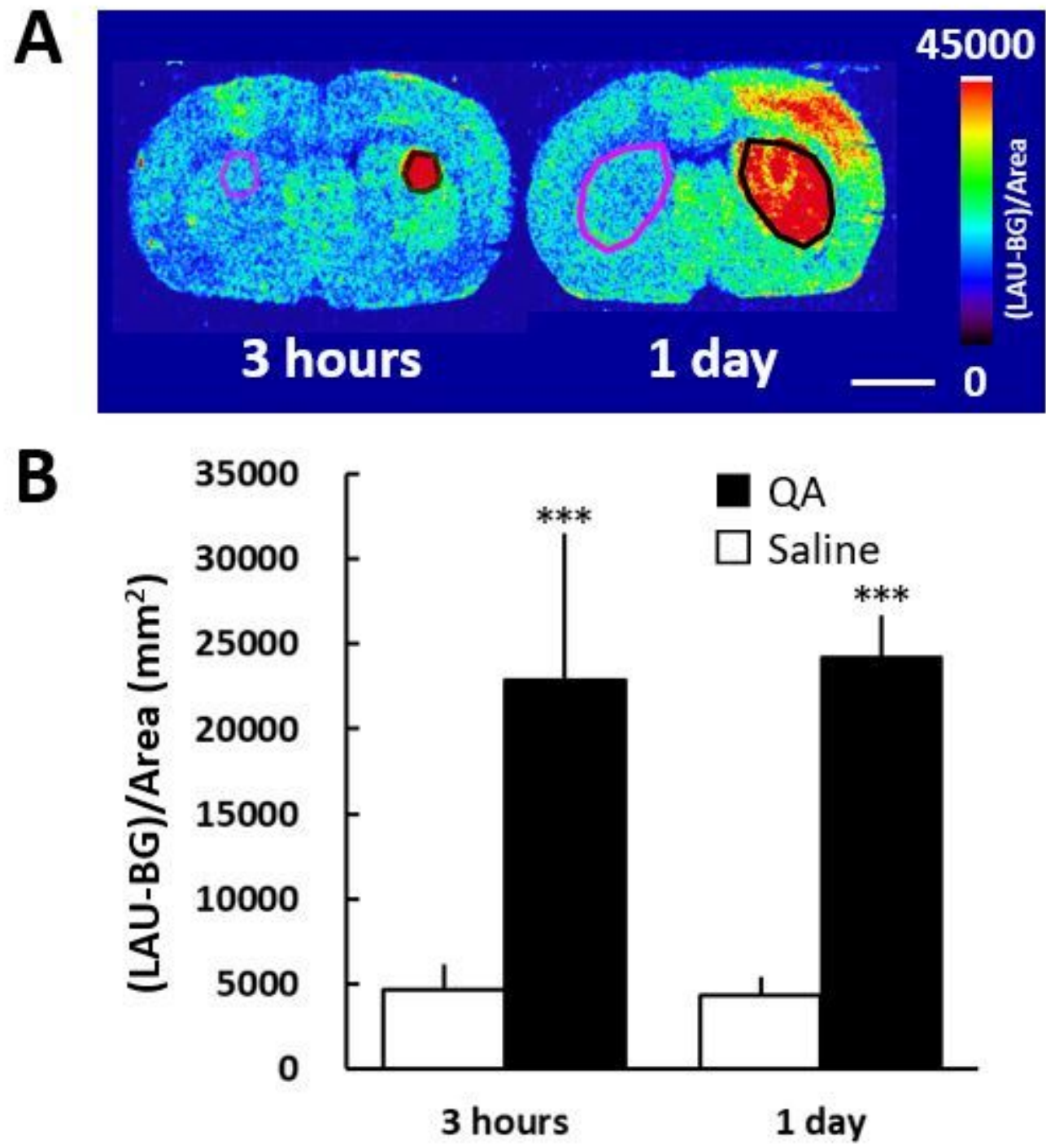
Figure 1

DHE uptake at 3 hours and 1 day after QA injection. QA was injected into the striatum on the right side of the image, and saline was simultaneously injected into the contralateral striatum. A; Accumulation of fluorescence in the QA-injected striatum 60 minutes after the intravenous injection of DHE. B;

Semiquantitative analysis of the fluorescent images. The regions of interest (ROIs) were identified based on the strong fluorescence signal region of the striatum and the corresponding region on the opposite side. Mean $\pm S D, n=4-9$. ${ }^{*} * \mathrm{P}<0.001$, between the saline- and the QA-injected side using the Student's paired t-test. Bar $=5 \mathrm{~mm}$

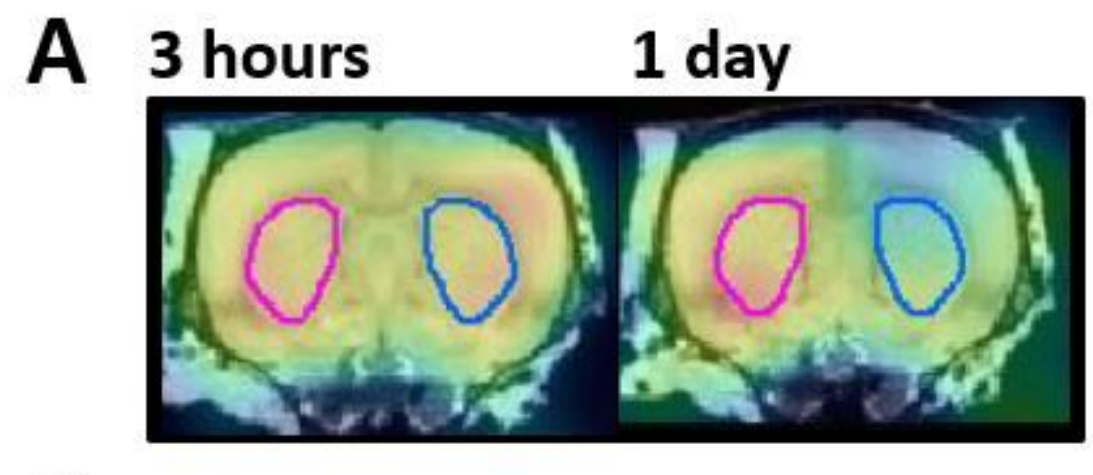

B

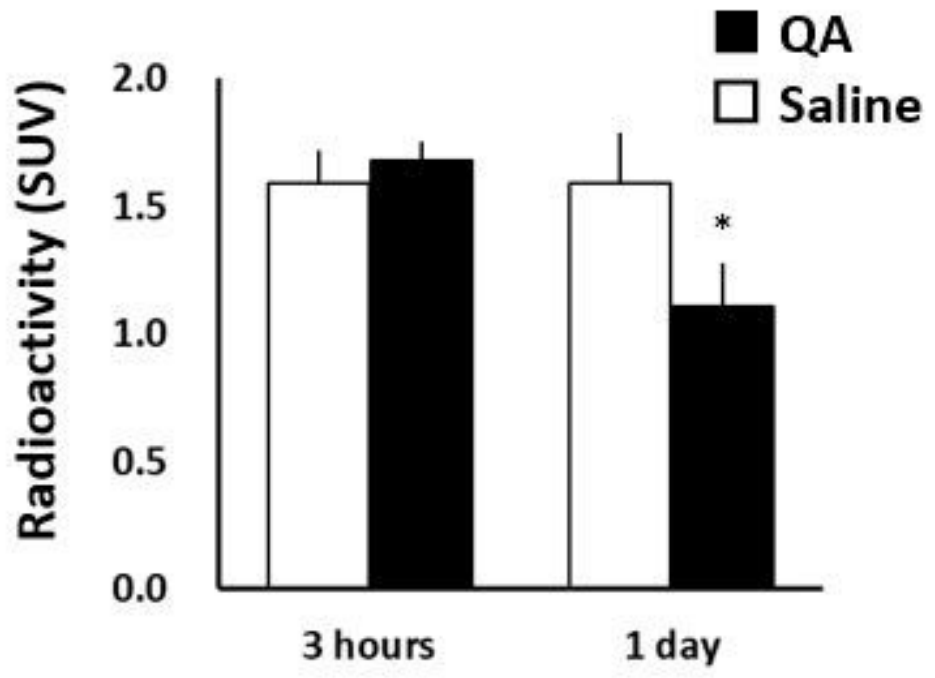

\section{Figure 2}

18F-BCPP-EF uptake at 3 hours and 1 day after QA injection. QA was injected into the striatum on the right side of the image, and saline was simultaneously injected into the contralateral striatum. A; PET images of 18F-BCPP-EF uptake in QA-injected rat brains were superimposed on the MRI atlas images. PET scans were performed for 90 minutes with 18F-BCPP-EF, 3 hours and 1 day after QA injection. 
Summation PET images from 45 to 60 minutes were reconstructed to obtain standardised uptake value (SUV) images. B; Radioactivity concentrations for the time period from 45-60 minutes (SUV). The VOls were identified based on the whole striatum. Mean $\pm S D, n=4$. ${ }^{*}<0.05$, between the saline- and QAinjected sides using the Student's paired t-test

\section{Bregma}

\section{$3.2 \mathrm{~mm} \quad-3.3 \mathrm{~mm} \quad-12.3 \mathrm{~mm}$}

\section{A DHE}

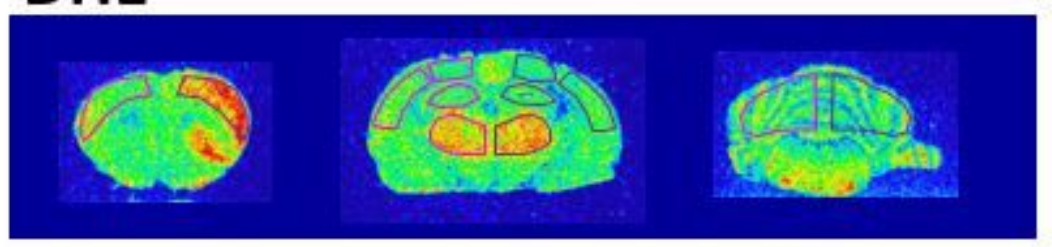

B ${ }^{18} \mathrm{~F}-\mathrm{BCPP}-\mathrm{EF}$
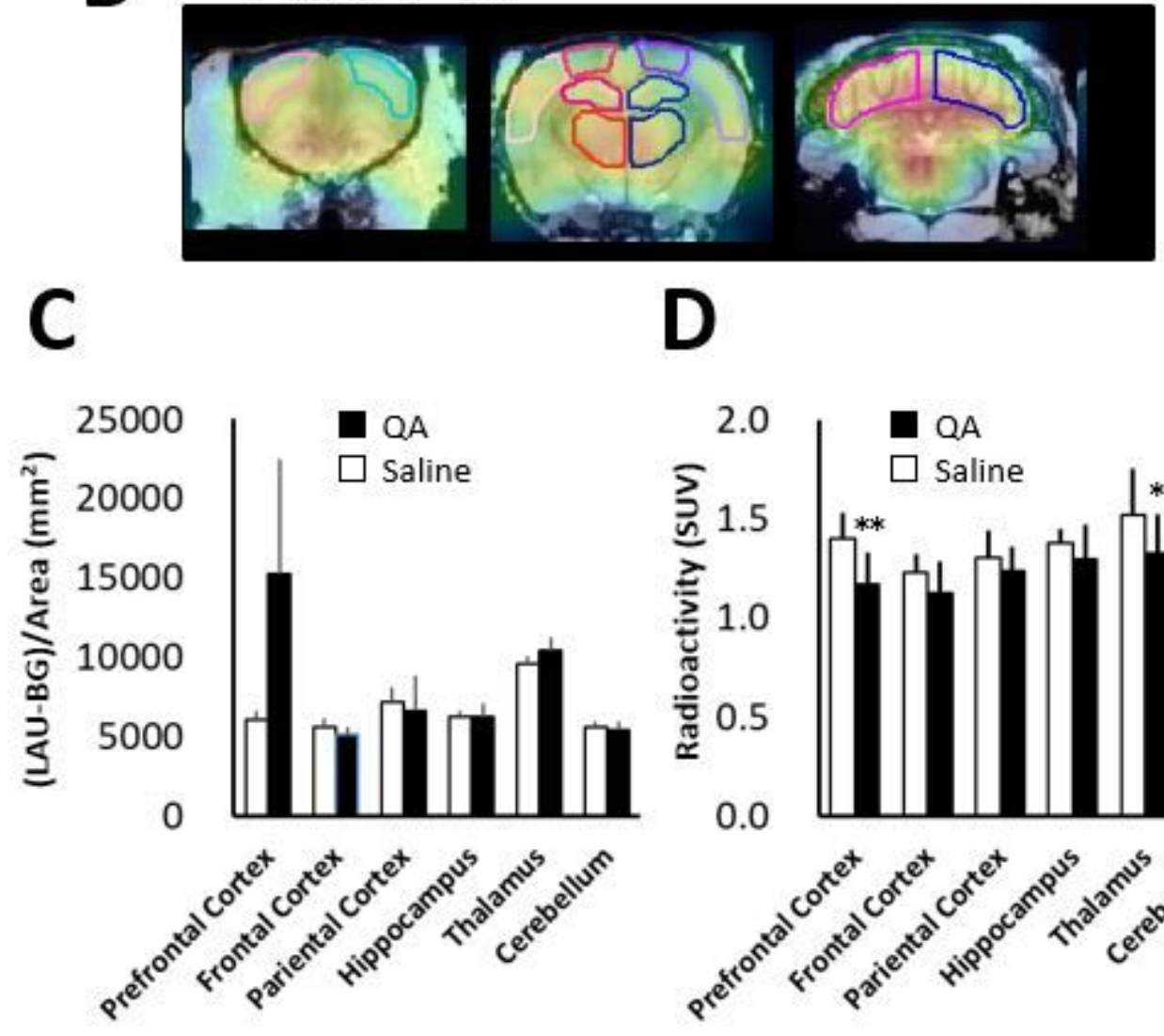

D

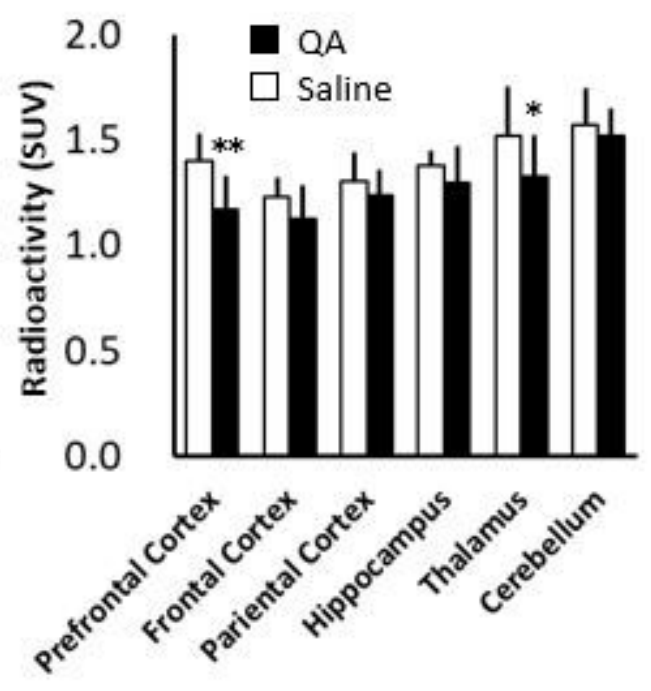

Figure 3

DHE and 18F-BCPP-EF uptake in various brain regions 1 day after QA injection. QA was injected into the striatum on the right side of the image, and saline was simultaneously injected into the contralateral striatum. A; The accumulation of fluorescence in the QA-injected striatum 60 minutes after the 
intravenous injection of DHE. B; Summation 18F-BCPP-EF PET images from 45 to 60 minutes were reconstructed to obtain standardised uptake value (SUV) images. C; Semiquantitative analysis of the fluorescent images. The ROls were identified based on the prefrontal cortex, frontal cortex, parietal cortex, hippocampus, thalamus, and cerebellum. Mean $\pm S D, n=4$. D; Radioactivity concentration for the time period from 45-60 minutes (SUV). The VOIs were identified based on the prefrontal cortex, frontal cortex, parietal cortex, hippocampus, thalamus, and cerebellum. Mean $\pm S D, n=4$. ${ }^{*} P<0.05,{ }^{*} P<0.01$ between the saline- and the QA-injected side using the Student's paired t-test

\section{QA \\ 3 hours $B$}

saline

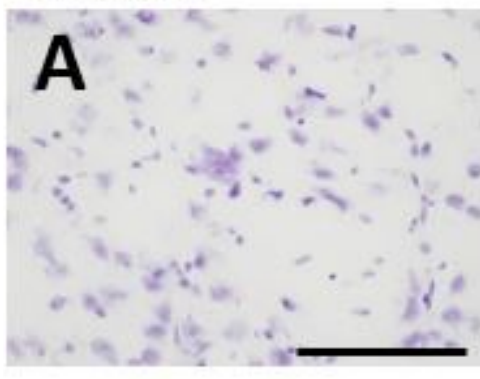

saline

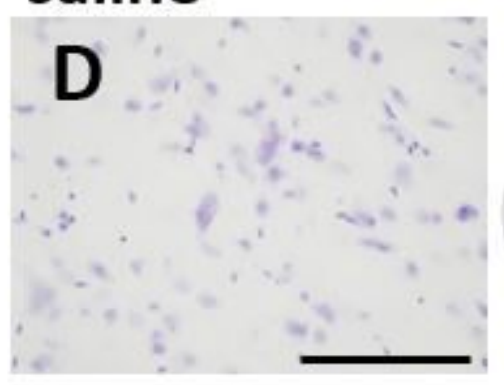

1 day

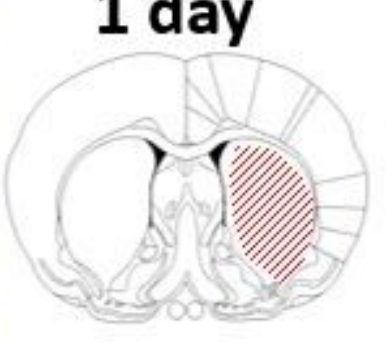

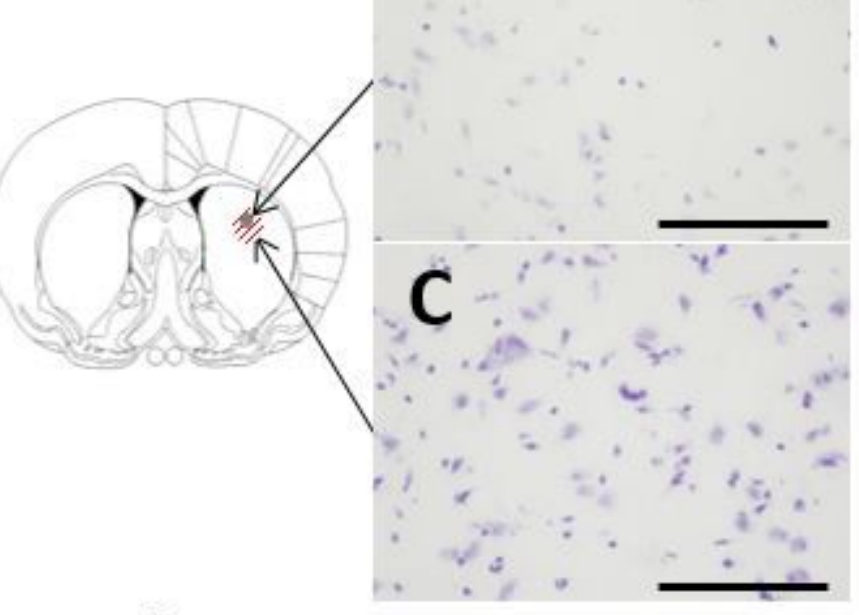

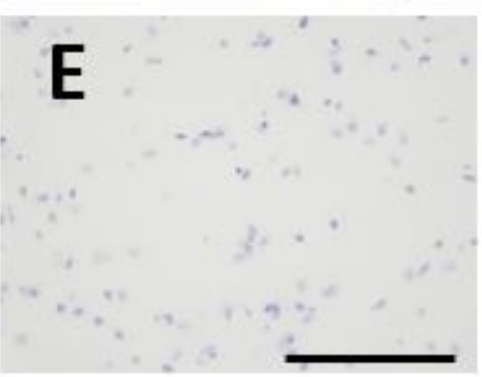

\section{Figure 4}

Nissl-stained brain slices within the striatum after the acquisition of ex vivo fluorescent images. QA was injected into the striatum, and saline was simultaneously injected into the contralateral striatum. Three hours $(A-C)$ or 1 day (D and $E$ ) after QA or saline injection. Neuronal degradation was observed in the greyed-out area within an approximately 1.0-mm-diameter area (B) and in the whole striatum (E). The red hatched area corresponds to the area of increased fluorescence signal. The images in A, C, and D showed no abnormalities. Bar $=100 \mu \mathrm{m}$ 


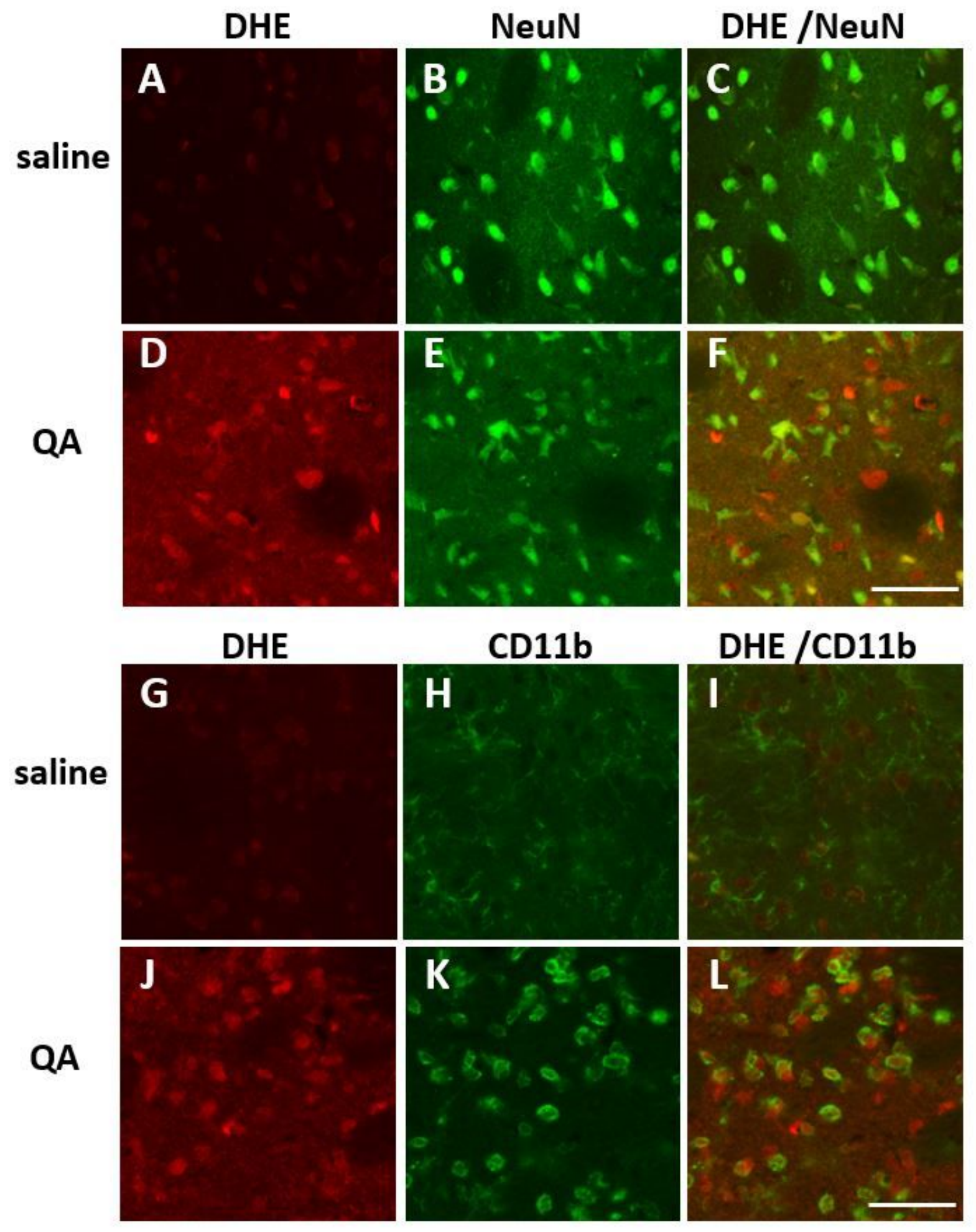

Figure 5

Immunohistochemical analysis of NeuN- or CD11b-positive cells in the saline- or QA-injected rat striatum. Saline or QA was injected into the striatum 1 day before DHE administration. Bar $=50 \mu \mathrm{m}$

\section{Supplementary Files}


This is a list of supplementary files associated with this preprint. Click to download.

- QA20210423SupplFigureHosoi.pptx 